\title{
IDEALS AND OTHER GENERALIZATIONS OF CONGRUENCE CLASSES
}

\author{
PAOLO AGLIANO and ALDO URSINI
}

(Received 16 October 1990)

Communicated by T. E. Hall

\begin{abstract}
In the general context of ideals in universal algebras, we study varietal properties connected with ideals that are equivalent both to $\mathrm{Mal}^{\prime}$ cev conditions and congruence properties such as 0-regularity, 0-permutability, etc.
\end{abstract}

1991 Mathematics subject classification (Amer. Math. Soc.): 08 A 30, 08 B 99.

This paper continues the investigations of the authors on ideals in general algebras (see [1], [2], [3], [5], [9], [10], [11]). The general program of this series of papers can be roughly described as follows.

Let $\mathbf{A}$ be an algebra in a variety with a distinguished element 0 . A sequence of successively stronger closure operators is defined on $\mathbf{A}$ in such a way that there is a natural map $\varphi$ from the congruence lattice of $\mathbf{A}$ (or some other "interesting" sublattice of $\mathbf{A}^{2}$ ) to the lattice of closed subsets of $A$ containing 0 . Some natural questions are:

(1) When is $\varphi$ one-to-one?

(2) When is $\varphi$ onto?

(3) When is $\varphi$ a homomorphism?

These questions are usually asked in the context of varieties. The object is to find $\mathrm{Mal}^{\prime} \mathrm{cev}$ conditions for these properties and characterizations in terms of congruence properties such as permutabilty, 0-permutability, etc. Theorem 2.4 is one example of the type of result desired.

Our notation is more or less standard; we use the notation a for $a_{1}, \ldots, a_{n}$ (C) 1992 Australian Mathematical Society 0263-6115/92 \$A2.00+0.00 
and unless necessary we will write $\mathbf{a} \in A$ for $\mathbf{a} \in A^{n}$. For general background in universal algebra we refer to [7].

\section{Introduction}

As we pointed out above all algebras and varieties considered in this paper belong to a type with a nullary operation (or have a definable constant) denoted by 0 .

Definition 1.1. Given an algebra $\mathrm{A}$, a nonempty subset $H$ of $\mathrm{A}$ is a clot of $\mathbf{A}$ if

(1) $0 \in H$.

(2) For all positive integers $n$, for all $f\left(x_{1}, \ldots, x_{n}\right) \in \operatorname{Pol}_{n}(\mathbf{A})$ with $f(0, \ldots, 0)=0$ we have $f\left(h_{1}, \ldots, h_{n}\right) \in H$ for all $h_{1}, \ldots, h_{n} \in H$.

DEFINITION 1.2. Let $\mathscr{V}$ be a variety of algebras.

(1) A $\mathscr{V}$-ideal term in $\mathbf{y}$ [4] is a term $t(\mathbf{x}, \mathbf{y})$ such that

$$
t(\mathbf{x}, 0, \ldots,) \approx 0
$$

holds in $\mathscr{V}$.

(2) Given an algebra $\mathbf{A} \in \mathscr{V}$, a subset $I$ of $A$ is a $\mathscr{V}$-ideal of $\mathbf{A}$ if for any $\mathscr{V}$-ideal term $t(\mathbf{x}, \mathbf{y})$ in $\mathbf{y}$ and for any $a_{1}, \ldots, a_{m} \in A, b_{1}, \ldots, b_{n} \in I$ we have

$$
t\left(a_{1}, \ldots, a_{m}, b_{1}, \ldots, b_{n}\right) \in I .
$$

If $\mathscr{V}=\boldsymbol{V}(A)$ then we will write ideal term and ideal instead of $\mathscr{V}$-ideal term and $\mathscr{V}$-ideal. Some other notations ought to be established right away: as usual $\operatorname{Con}(A)$ denotes the congruence lattice of $\mathbf{A}$ and for $X \subseteq A, \vartheta(X)$ denotes the congruence generated by $X \times X$. Moreover

(1) $\mathrm{Cl}(\mathbf{A})$ is the set of all the clots of $\mathbf{A}$,

(2) $\mathbf{I}(A)$ is the set of all the ideals of $\mathbf{A}$,

(3) $\mathrm{N}(\mathbf{A})=\{0 / \theta: \theta \in \operatorname{Con}(\mathbf{A})\}$ and the sets in $\mathrm{N}(\mathbf{A})$ are called normal sets,

(4) For $X \subseteq A$ and $S \subseteq A \times A, X / S=\{y:(x, y) \in S$ for some $x \in X\}$.

Each of $\mathrm{Cl}(\mathbf{A}), \mathrm{I}(\mathbf{A})$ and $\mathrm{N}(\mathbf{A})$ is an algebraic lattice, where the meet is the set intersection and the join will be described shortly. The inclusion relation between them is easily seen to be

$$
\mathrm{N}(\mathbf{A}) \subseteq \mathrm{Cl}(\mathbf{A}) \subseteq \mathrm{I}(\mathbf{A}) .
$$

Note that we do not mean to imply that any of these inclusions is a sublattice inclusion. Trivial examples show that in general the inclusions are proper; however, if $\mathscr{V}$ is the variety of pointed sets, then $\mathrm{N}(\mathbf{A})=\mathbf{I}(\mathbf{A})$ for all $\mathbf{A} \in \mathscr{V}$. By the same token the equality of any two of these sets for all algebras in a variety is not a $\mathrm{Mal}^{\prime}$ cev condition. 
We now proceed to describe the join in the aforementioned lattices; namely, for $X \subseteq A$ we describe $\langle X\rangle_{\mathrm{N}},\langle X\rangle_{\mathrm{Cl}},\langle X\rangle_{\mathrm{I}}$, that are respectively the normal set, the clot and the ideal generated by $X$.

Proposition 1.3. (1) $\langle X\rangle_{\mathrm{N}}=0 / \vartheta(X \cup\{0\})$.

$$
\begin{aligned}
& \langle X\rangle_{\mathrm{Cl}}=\{t(\mathbf{a}, \mathbf{b}): t(\mathbf{a}, 0, \ldots, 0), \mathbf{a} \in A, \mathbf{b} \in X \cup\{0\}\} . \\
& \langle X\rangle_{\mathrm{I}}=\{t(\mathbf{a}, \mathbf{b}): t(\mathbf{x}, \mathbf{y}) \text { an ideal term in } \mathbf{y}, \mathbf{a} \in A, \mathbf{b} \in X\} .
\end{aligned}
$$

All proofs are simple exercises that are really consequences of the fact that the set of terms in consideration is, in each case, closed under composition.

In the sequel, for a given algebra $\mathbf{A}$, we will often deal with reflexive subalgebras of $\mathbf{A} \times \mathbf{A}$ and we will call them semicongruences of $\mathbf{A}$. It is clear that semicongruences form an algebraic lattice under inclusion, denoted by $\operatorname{Sc}(\mathbf{A})$. Moreover for any $X \subseteq A$ we define

$$
X^{*}=\text { the semicongruence of } \mathbf{A} \text { generated by }\{0\} \times X
$$

and we leave to the reader the easy task of checking that

$$
X^{*}=\text { the subalgebra of } \mathbf{A}^{2} \text { generated by } \operatorname{diag}(A) \cup\{0\} \times X .
$$

One might say that this paper is a series of comments to the following simple and neat result of $\mathrm{Mal}^{\prime} \mathrm{cev}$, implicit in his fundamental work [6].

Proposition 1.4. For any algebra $\mathbf{A}$ and for any $X \subseteq A$ the following are equivalent.

(1) $X \in \mathrm{N}(\mathbf{A})$.

(2) $X=0 / \vartheta(X)$.

(3) $0 \in X$ and for every unary polynomial $g(x)$ of $\mathbf{A}$, for any $a, b \in X$, if $g(a) \in X$ then $g(b) \in X$.

(4) $0 \in X$ and for every unary polynomial $g(x)$ of $\mathbf{A}$, for any $a \in X$, $g(a) \in X$ if and only if $g(0) \in X$.

\section{The theorems}

The first result is an analogue of Proposition 1.4 when one considers ideals instead of normal sets.

THEOREM 2.1. For any algebra $\mathbf{A}$ the following are equivalent.

(1) Every ideal is a congruence class, i.e. $\mathbf{I}(\mathbf{A})=\mathrm{N}(\mathbf{A})$.

(2) $\langle X\rangle_{\mathrm{I}}=0 / \vartheta(X)$ for any $X \subseteq A$.

(3) $I / \vartheta(J)=I \vee J$ for any $I, J \in \mathrm{I}(\mathbf{A})$. 
(4) $I / \vartheta(J)=J / \vartheta(I)$ for any $I, J \in \mathbf{I}(\mathbf{A})$.

(5) The mapping from $\mathbf{I}(\mathbf{A})$ to $\mathrm{Con}(\mathbf{A})$ sending $I \longmapsto \vartheta(I)$ is one-to-one.

(6) The mapping from $\operatorname{Con}(\mathbf{A})$ to $\mathbf{I}(\mathbf{A})$ sending $\theta \longmapsto 0 / \theta$ is onto.

Proof. (1), (2) and (6) are clearly equivalent. And (2) implies (5) since, if $I, J \in \mathbf{I}(\mathbf{A})$ and $\vartheta(I)=\vartheta(J)$, then $0 / \vartheta(I)=0 / \vartheta(J)$, hence, via (2), $I=J$. Assume now (5). We claim that, for any $I \in I(\mathbf{A})$, we have $\vartheta(I)=\vartheta(0 / \vartheta(I))$. One inclusion is obvious, since $I \subseteq 0 / \vartheta(I)$. Let then $(u, v) \in \vartheta(0 / \vartheta(I))$; by the congruence generation theorem, there exists a positive integer $n, a_{1}, \ldots, a_{n}, b_{1}, \ldots, b_{n} \in 0 / \theta(I)$ and binary polynomials $f_{1}, \ldots, f_{n}$ such that

$$
\begin{aligned}
u & =f\left(a_{1}, b_{1}\right) \\
f_{1}\left(b_{1}, a_{1}\right) & =f_{2}\left(a_{2}, b_{2}\right) \\
& \vdots \\
f_{n}\left(b_{n}, a_{n}\right) & =v .
\end{aligned}
$$

Since $a_{i}, b_{i} \in 0 / \vartheta(I)$ for all $i$, we have

$$
\begin{aligned}
u & =f_{1}\left(a_{1}, b_{1}\right) \vartheta(I) \varphi_{1}(0,0) \vartheta(I) f_{1}\left(b_{1}, a_{1}\right) \\
& =f_{2}\left(a_{2}, b_{2}\right) \vartheta(I) f_{2}(0,0) \vartheta(I) \ldots \vartheta(I) f_{n}\left(a_{n}, b_{n}\right)=v .
\end{aligned}
$$

So $(u, v) \in \vartheta(I)$ and the claim is proved. But via (5) this implies $I=0 / \theta(I)$ and hence (2).

Assume now (2). The left-to-right inclusion in (3) is easy, since $u \in I / \vartheta(J)$ implies that $(i, u) \in \vartheta(J)$ for some $i \in I$. But $0 \in I$, so $(0, i) \in \vartheta(I)$ and hence $(0, u) \in \vartheta(I) \vee \vartheta(J) \subseteq \vartheta(I \vee J)$. Hence, by (2) $u \in I \vee J$. On the other hand, if $u \in I \vee J$, then, by Proposition 1.3(5), there is an ideal term $t(\mathbf{x}, \mathbf{y}), \mathbf{a} \in A, \mathbf{i} \in I$ and $\mathbf{j} \in J$, such that $u=t(\mathbf{a}, \mathbf{i}, \mathbf{j})$. But then

$$
u=t(\mathbf{a}, \mathbf{i}, \mathbf{j}) \vartheta(J) t(\mathbf{a}, \mathbf{i}, 0, \ldots, 0) \in I .
$$

Therefore $u \in I / \vartheta(J)$ and the other inclusion is proved.

That (3) implies (4) is obvious. On the other hand if in the equality in (4) we set $J=\langle 0\rangle_{I}=\{0\}$ we get at once $I=I / \vartheta(\{0\})=0 / \vartheta(I)$. Hence (4) implies (2) and the proof is finished.

The next step is to show that semicongruences are, in some sense, the natural correspondent of congruences for clots.

Proposition 2.2. Let $\mathbf{A}$ be an algebra and $X \subseteq A$. Then conditions (1), (2) and (3) are equivalent.

(1) $X$ is a clot. 
(2) $X=0 / S$ for some $S \in \mathrm{Sc}(\mathbf{A})$.

(3) $X=0 / X^{*}$.

Moreover,

(4) If $X, Y \subseteq A$, we have $0 / X^{*}=0 / Y^{*}$ if and only if $X^{*}=Y^{*}$.

(5) If $X, Y \in \mathrm{Cl}(\mathbf{A})$, then $X=Y$ if and only if $X^{*}=Y^{*}$. In other words the mapping from $\mathrm{Cl}(\mathbf{A})$ to $\mathrm{Sc}(\mathbf{A})$ defined by $X \longmapsto X^{*}$ is injective.

Proof. First note that, if $S \in \operatorname{Sc}(\mathbf{A})$, then $0 / S$ is always a clot. In fact if $f$ is an $n$-ary polynomial such that $f(0, \ldots, 0)=0$ and $a_{1}, \ldots, a_{n} \in 0 / S$ then

$$
\begin{aligned}
\left\langle 0, f\left(a_{1}, \ldots, a_{n}\right)\right) & =\left(f(0, \ldots, 0), f\left(a_{1}, \ldots, a_{n}\right)\right\rangle \\
& =f\left(\left\langle 0, a_{1}\right\rangle, \ldots,\left\langle 0, a_{n}\right\rangle\right) \in S
\end{aligned}
$$

since $\mathbf{S}$ is a reflexive subalgebra of $\mathbf{A}^{2}$. Hence $f\left(a_{1}, \ldots, a_{n}\right) \in 0 / S$. We have thus proved that $0 / S$ is a clot.

Suppose now that $X$ is a clot; clearly $X \subseteq 0 / X^{*}$. Let $a \in 0 / X^{*}$; by definition of $X^{*}$ there is a term $t(\mathbf{x})$ and $a_{1}, \ldots, a_{n}, b_{1}, \ldots, b_{m} \in A$ such that

$$
\begin{aligned}
\langle 0, a\rangle & =t\left(\left\langle a_{1}, a_{1}\right\rangle, \ldots,\left\langle a_{n}, a_{n}\right\rangle,\left\langle 0, b_{1}\right\rangle, \ldots,\left\langle 0, b_{m}\right\rangle\right) \\
& =\left\langle t\left(a_{1}, \ldots, a_{n}, 0, \ldots, 0\right), t\left(a_{1}, \ldots, a_{n}, b_{1}, \ldots, b_{m}\right)\right\rangle .
\end{aligned}
$$

Let now $f$ be the $m$-ary polynomial of $\mathbf{A}$ defined by

$$
f\left(x_{1}, \ldots, x_{m}\right)=t\left(a_{1}, \ldots, a_{n}, x_{1}, \ldots, x_{m}\right) .
$$

Then from the equalities above $f(0, \ldots, 0)=0$ so, $X$ being a clot, $f\left(b_{1}, \ldots, b_{m}\right) \in X$. But

$$
a=t\left(a_{1}, \ldots, a_{n}, b_{1}, \ldots, b_{m}\right)=f\left(b_{1}, \ldots, b_{m}\right) \in X,
$$

and hence $X=0 / X^{*}$. This proves the equivalence of (1), (2) and (3).

For (4) we assume $0 / X^{*}=0 / Y^{*}$, the other implication being trivial. Let $\langle a, b\rangle \in X^{*}$. Then, exactly as above, we can find a term $t, a_{1}, \ldots, a_{n} \in A$ and $c_{1}, \ldots, c_{m} \in X$, such that

$$
t\left(a_{1}, \ldots, a_{n}, 0, \ldots, 0\right)=a \quad t\left(a_{1}, \ldots, a_{n}, c_{1}, \ldots, c_{m}\right)=b .
$$

But since $\left\langle 0, c_{i}\right\rangle \in X^{*}$ for all $i \leq m$ the hypothesis yields $\left\langle 0, c_{i}\right\rangle \in Y^{*}$ and therefore $\langle a, b\rangle \in Y^{*}$, the latter being a reflexive subalgebra of $\mathbf{A}^{2}$. Hence $X^{*} \subseteq Y^{*}$, while the reverse inclusion can be obtained with a symmetric argument. Therefore (4) holds. Finally (5) is an easy consequence of (3) and (4). 
Note that we are not saying that the $S$ in the above proposition is unique and easy examples show that this is not always the case. As a matter of fact, asking that to hold for a whole variety is a rather strong request, as shown in Theorem 2.7 below.

Let now $S \in \operatorname{Sc}(\mathbf{A})$. We say that $S$ is 0 -symmetric if $\langle 0, x\rangle \in S$ if and only if $\langle x, 0\rangle \in S$. We say that $S$ is 0 -transitive if $\langle 0, x\rangle \in S$ and $\langle x, y\rangle \in S$ imples $\langle 0, y\rangle \in S$. If a semicongruence is both 0 -symmetric and 0 -transitive then it behaves "locally" like a congruence. The impact of this behavior on clot is displayed in the proposition below.

Proposition 2.3. Let $\mathbf{A}$ be an algebra. If every semicongruence of $\mathbf{A}$ is 0-symmetric and 0-transitive, then $\mathrm{N}(\mathbf{A})=\mathrm{Cl}(\mathbf{A})$.

Proof. Let $X \in \mathrm{Cl}(\mathbf{A})$. Then $X=0 / X^{*}$. We show that if $X^{*}$ is 0 symmetric then it is in fact symmetric. Suppose that $\langle a, b\rangle \in X^{*}$. Then as in the proof of Proposition 2.2(4) there exist a term $t, \mathbf{a} \in A$ and $b_{1}, \ldots, b_{n} \in$ $X$ with

$$
t(\mathbf{a}, 0, \ldots, 0)=a, \quad t\left(\mathbf{a}, b_{1}, \ldots, b_{n}\right)=b .
$$

Since $\left\langle 0, b_{i}\right\rangle \in X^{*}$ for all $i \leq n, 0$-symmetricity yields $\left\langle b_{i}, 0\right\rangle \in X^{*}$ for all $i \leq n$ and therefore

$$
\langle b, a\rangle=\left\langle t\left(\mathbf{a}, b_{1}, \ldots, b_{n}\right), t(\mathbf{a}, 0, \ldots, 0)\right\rangle \in X^{*} .
$$

Hence $X^{*}$ is symmetric. Let now $\theta=\vartheta(\{\langle 0, x\rangle: x \in X\})$. It is clear that $\theta$ is the transitive closure of $X^{*}$, and hence, if $y \in 0 / \theta$ there exist $y_{0}=0, y_{1}, \ldots, y_{k}=y$ such that $\left\langle y_{i}, y_{i+1}\right\rangle \in X^{*}$. It is now obvious that 0 transitivity yields $\langle 0, y\rangle \in X^{*}$. Hence $0 / \theta \subseteq 0 / X^{*}$, and the other inclusion being trivial, we may conclude $X=0 / X^{*}=0 / \theta$. Therefore $X \in \mathrm{N}(A)$ and the proof is finished.

Let us point out that the implication in the above proposition cannot be reversed, where again pointed sets give a counterexample.

If we ask the hypothesis of the above proposition to hold for any algebra in a whole variety, then again the request is much stronger and not surprisingly it turns out that 0 -symmetricity and 0 -transitivity are closely related to 0 permutability. Let us recall [5] that two congruences $\theta, \varphi$ are said to permute at 0 if $\langle 0, x\rangle \in \theta \vee \varphi$ implies $\langle 0, y\rangle \in \theta$ and $\langle x, y\rangle \in \varphi$ for some $y$. This is clearly equivalent to saying that if $\langle 0, x\rangle \in \theta \circ \varphi$ then $\langle 0, x\rangle \in \varphi \circ \theta$.

THEOREM 2.4. For a variety $\mathscr{V}$ the following are equivalent.

(1) For all $\mathbf{A} \in \mathscr{V}$ and $\theta, \varphi \in \operatorname{Con}(\mathbf{A})$ we $0 /(\theta \vee \varphi)=0 /(\theta \circ \varphi)$

(2) Every algebra in $\mathscr{V}$ has 0-permutable congruences. 
(3) There is a binary term $s(x, y)$ of $\mathscr{V}$ such that

$$
s(x, x) \approx 0, \quad s(x, 0) \approx x
$$

hold in $\mathscr{V}$.

(4) There is a ternary term $w(x, y, w)$ of $\mathscr{V}$ such that

$$
w(x, y, y) \approx x, \quad w(x, x, 0) \approx 0
$$

hold in $\mathscr{V}$.

(5) There exists a positive integer $m$, binary terms $d_{1}(x, y), \ldots, d_{m}(x, y)$ and an $m+3$-ary term $q\left(x_{1}, \ldots, x_{m+3}\right)$ of $\mathscr{V}$ such that

$$
\begin{aligned}
& d_{i}(x, x) \approx 0 \text { for } i=1, \ldots, m \\
& q(x, y, 0,0, \ldots, 0) \approx 0 \\
& q\left(x, y, y, d_{1}(x, y), \ldots, d_{m}(x, y)\right) \approx x
\end{aligned}
$$

hold in $\mathscr{V}$.

(6) $\mathscr{V}$ is ideal-coherent, that is, for all $\mathbf{A} \in \mathscr{V}, I \in \mathbf{I}(\mathbf{A})$, if $0 / \theta \subseteq I$ for some $\theta \in \operatorname{Con}(\mathbf{A})$, then $I$ is a union of $\theta$-blocks.

(7) For all $\mathbf{A} \in \mathscr{V}$, the mapping $\operatorname{Con}(\mathbf{A}) \longrightarrow \mathbf{I}(\mathbf{A})$ defined by $\theta \longmapsto 0 / \theta$ is a complete and onto lattice homomorphism.

(8) For all $\mathbf{A} \in \mathscr{V}$, every semicongruence of $\mathbf{A}$ is 0-symmetric and 0 -transitive.

(9) For all $\mathbf{A} \in \mathscr{V}$, every semicongruence of $\mathbf{A}$ is 0-transitive.

(10) The semicongruences of any algebra in $\mathscr{V}$ permute at 0.

Proof. That (1), (2), (3) and (4) are equivalent has been proved explicitly or implicitly in [5]. Moreover (3) implies (5) if one puts $m=1, d_{1}(x, y)=$ $s(x, y)$ and $q(x, y, z, w)=s(x, s(s(x, z), w))$.

Assume then (5). Let $I \in \mathbf{I}(\mathbf{A}), \theta \in \operatorname{Con}(\mathbf{A})$ and $0 / \theta \subseteq I$. Let $v \in I$ with $\langle u, v\rangle \in \theta$; then for all $i \leq m$ we have $d_{i}(u, v) \theta d_{i}(v, v)=0$, and hence $d_{i}(u, v) \in I$. Note that $q(x, y, z)$ is an ideal term in $\mathbf{z}$, so we must have

$$
u=q\left(u, v, v, d_{1}(u, v), \ldots, d_{m}(u, v)\right) \in I .
$$

Hence (5) implies (6). For the converse, assume that $\mathscr{V}$ is ideal-coherent and look at $\mathbf{F}_{\mathscr{V}}(x, y)$. Let $\theta_{f}$ be the congruence associated with the endomorphism of $\mathbf{F}_{\mathscr{V}}(x, y)$ defined by $f(x)=f(y)=x$ and $f(0)=0$. Let

$$
I=\left\langle\{y\} \cup\left\{d(x, y) \in \mathbf{F}_{\mathscr{V}}(x, y): d(x, y) \in 0 / \theta_{f}\right\}\right\rangle_{\mathbf{I}} .
$$

Then clearly $0 / \theta_{f} \subseteq I, y \in I$ and $\langle x, y\rangle \in \theta_{f}$. Thus ideal-coherency yields $x \in I$. By Proposition 1.3(4), there is an ideal term $t(\mathbf{x}, y, \mathbf{z})$ in $y \cup \mathbf{z}$ and $d_{1}(x, y), \ldots, d_{m}(x, y) \in 0 / \theta_{f}$ with

$$
t\left(\mathbf{u}, y, d_{1}(x, y), \ldots, d_{m}(x, y)\right)=x .
$$


Since any $u_{j}=u_{j}(x, y)$ we do get an $(m+3)$-ary term by setting

$$
q\left(x, y, y, z_{1}, \ldots, z_{m}\right)=t\left(\mathbf{u}, y, z_{1}, \ldots, z_{m}\right) .
$$

But then $q(x, y, 0,0, \ldots, 0)=0$, since $q$ is an ideal term in $y \cup z$. As shown above $q\left(x, y, y, d_{1}(x, y), \ldots, d_{m}(x, y)\right)=x$ and finally, for all $i$, $d_{i}(x, y) \in 0 / \theta_{f}$, which yields $d_{i}(x, x)=f\left(d_{i}(x, y)\right)=f(0)=0$. Therefore (5) and (6) are equivalent.

Assume again (5). Let $\mathbf{A} \in \mathscr{V}, \theta, \varphi \in \operatorname{Con}(\mathbf{A})$ and $a \in 0 /(\theta \circ \varphi)$. Then there is a $b \in A$ with $\langle 0, b\rangle \in \theta$ and $\langle b, a\rangle \in \varphi$. Hence we get $d_{i}(a, b) \varphi d_{i}(b, b) \varphi 0$ for all $i$. So

$$
\begin{aligned}
0= & q(a, b, 0,0, \ldots, 0) \\
& \varphi q\left(a, b, 0, d_{1}(a, b), \ldots, d_{m}(a, b)\right) \\
& \theta q\left(a, b, b, d_{1}(a, b), \ldots, d_{m}(a, b)\right)=a,
\end{aligned}
$$

and hence $\langle 0, a\rangle \in \varphi \circ \theta$ and $\mathscr{V}$ is 0 -permutable. Therefore (5) implies (2).

Assume now (3). Let $\mathbf{A} \in \mathscr{V}, \theta, \varphi \in \operatorname{Con}(\mathbf{A})$ and $a \in 0 /(\theta \vee \varphi)$. Then there are $a_{1}, \ldots, a_{n} \in A$ with

$$
a \theta a_{1} \varphi \alpha_{2} \theta \ldots \theta a_{n} \varphi 0 .
$$

Let us set $t(x, y, z)=,s(x, s(s(x, y), z)$,$) and let us induct on n$. If $n=1$ then $a \theta a_{1} \varphi 0$. Hence $s\left(a, a_{1}\right) \theta 0$, therefore

$$
a=t\left(a, a_{1}, s\left(a, a_{1}\right)\right) \in 0 /(\theta \vee \varphi),
$$

$t(x, y, z)$ being an ideal term in $y, z$. Let us now assume the statement true for $n$ and let

$$
a \theta a_{1} \varphi \alpha_{2} \theta \ldots \theta a_{n} \varphi a_{n+1} \theta 0 .
$$

Then $s\left(a, a_{n+1}\right) \varphi s\left(a, a_{n}\right) \theta \ldots \theta s(a, a)=0$, so, by the induction hypothesis, $s\left(a, a_{n+1}\right) \in 0 / \theta \vee 0 / \varphi$. But since $a_{n+1} \in 0 / \theta$ we get again

$$
a=t\left(a, a_{n+1}, s\left(a, a_{n+1}\right)\right) \in 0 / \theta \vee 0 / \varphi \text {. }
$$

The case $a_{n+1} \varphi 0$ is totally similar and hence we conclude that $0 /(\theta \vee \varphi) \subseteq$ $0 / \theta \vee 0 / \varphi$. For the converse let $a \in 0 / \theta \vee 0 / \varphi$; then there is an ideal term $p(\mathbf{x}, \mathbf{y}, \mathbf{z})$ in $\mathbf{y} \cup \mathbf{z}, \mathbf{a} \in A, \mathbf{u} \in 0 / \theta$ and $\mathbf{v} \in 0 / \varphi$ with $a=p(\mathbf{a}, \mathbf{u}, \mathbf{v})$. If we now set $b=p(\mathbf{a}, \mathbf{u}, 0, \ldots, 0)$ we get $\langle b, 0\rangle \in \theta$ and $\langle a, b\rangle \in \varphi$. Therefore $a \in 0 /(\theta \vee \varphi)$. We have thus proved that the mapping is a homomorphism. Let now $I \in I(\mathbf{A})$; we will prove that it is a congruence class by using the "Mal'cev criterion" (Proposition 1.4). Let then $a, b \in I$, and let $g(x)$ be a unary polynomial of $\mathbf{A}$ and assume that $g(a) \in I$. Clearly $g(x)=$ $t\left(a_{1}, \ldots, a_{n}, x\right)$ for some term $t$ and $a_{1}, \ldots, a_{n} \in A$. Now

$$
w\left(y_{1}, t\left(x_{1}, \ldots, x_{n}, y_{2}\right), t\left(x_{1}, \ldots, x_{n}, y_{3}\right)\right)
$$


is an ideal term in $y_{1}, y_{2}, y_{3}$. Therefore

$$
c=w\left(g(b), t\left(a_{1}, \ldots, a_{n}, a\right), t\left(a_{1}, \ldots, a_{n}, a\right)\right) \in I .
$$

But $c=w(g(b), g(a), g(a))=g(b)$. Hence $g(b) \in I$ and we conclude that $I$ is a block of a congruence. Therefore (3) implies (7).

That (7) implies (2) follows from the fact that $a \in 0 /(\theta \vee \varphi)$ implies $a \in 0 / \theta \vee 0 / \varphi$, hence $\langle a, 0\rangle \in \theta \circ \varphi$.

Assume now (4). Let $\mathbf{A} \in \mathscr{V}$ and $S \in \operatorname{Sc}(\mathbf{A})$. If $\langle 0, a\rangle \in S$, then $\langle 0,0\rangle,\langle a, a\rangle \in S$ as well, so

$$
\langle a, 0\rangle=\langle w(a, 0,0), w(a, a, 0)\rangle=w(\langle a, a\rangle,\langle 0, a\rangle,\langle 0,0\rangle) \in S
$$

since $\mathbf{S}$ is a subalgebra of $\mathbf{A}^{2}$. Similarly, if $\langle a, 0\rangle \in S$ we get $\langle 0, a\rangle \in S$, and hence $S$ is 0 -symmetric. Next if $\langle 0, a\rangle,\langle a, b\rangle \in S$ then

$$
\langle 0, b\rangle=\langle w(a, a, 0), w(b, a, a)\rangle=w(\langle a, b\rangle,\langle a, a\rangle,\langle 0, a\rangle) \in S
$$

so we conclude that $S$ is 0 -transitive as well and (4) implies (8). Next (8) implies (9) a fortiori. Assume (9) and let $S$ be the semicongruence generated by $\{\langle y, y\rangle,\langle y, x\rangle,\langle 0, y\rangle\}$ in $\mathbf{F}_{\mathscr{V}}(x, y)$. Since $S$ is 0 -transitive we must have $\langle 0, x\rangle \in S$. From here a standard Mal' cev argument yields the existence of a ternary term $w(x, y, z)$ satisfying the desired identities. Hence (9) and (4) are equivalent.

Finally it is clear that (10) implies (2). On the other hand assume (4) and let $S, R \in \operatorname{Sc}(\mathbf{A}),\langle 0, a\rangle \in S \circ R$. This implies the existence of $b \in A$ with $\langle 0, b\rangle \in S$ and $\langle b, a\rangle \in R$. But then

$$
0=w(b, b, 0) R w(a, b, 0) S w(a, b, b)=a,
$$

where we have used only the reflexivity of $R$ and $S$. Hence (4) implies (10) and the proof is finished.

Via Proposition 2.3 , if $\mathscr{V}$ is a variety satisfying any of the equivalent statements of Theorem 2.4, then for any $\mathbf{A} \in \mathscr{V}$ we have $\mathrm{N}(\mathbf{A})=\mathrm{Cl}(\mathbf{A})$. Note that we had no hope of finding the latter as an equivalent condition in the theorem above, since we have already seen that it cannot be expressed by a Mal'cev condition.

We have pointed out that, given an algebra $\mathbf{A}$, the normal set $0 / \theta$ is an ideal for any $\theta \in \operatorname{Con}(\mathbf{A})$ and the set $0 / S$ is a clot for any $S \in \operatorname{Sc}(\mathbf{A})$. It is only natural to ask what happens if any of these correspondences is oneto-one and onto for all the algebras in a variety and it is not a surprise that this property is related to another congruence property, 0-regularity. We say that an algebra $\mathbf{A}$ has 0-regular congruences [4] if for any $\theta, \varphi \in \operatorname{Con}(\mathbf{A})$, $0 / \theta=0 / \varphi$ implies $\theta=\varphi$. The next two theorems show the equivalence of 
any of the properties above to several statements. In particular any of them is expressible by a Mal'cev condition. The case of ideals has already been treated in [5] and we refer to that paper for the proof of Theorem 2.6 below.

Definition 2.5. Let $\mathscr{V}$ be a variety.

(1) [5] $\mathscr{V}$ is ideal-determined if, for all $\mathbf{A} \in \mathscr{V}, I \in \mathrm{I}(\mathbf{A})$, there is exactly one $\theta \in \operatorname{Con}(\mathbf{A})$ with $I=0 / \theta$.

(2) $\mathscr{V}$ is clot-determined if for all $\mathbf{A} \in \mathscr{V}, X \in \mathrm{Cl}(\mathbf{A})$ there is exactly one $S \in \operatorname{Sc}(\mathbf{A})$ such that $X=0 / S$.

THEOREM 2.6 [5]. For a variety $\mathscr{V}$ the following are equivalent.

(1) $\mathscr{V}$ is ideal determined.

(2) Any algebra in $\mathscr{V}$ has 0-regular and 0-permutable congruences.

(3) There exist a natural number $m$, binary terms $d_{1}(x, y), \ldots, d_{m}(x, y)$ and an $(m+3)$-term $q$ such that

$$
\begin{aligned}
& d_{i}(x, y) \approx 0 \quad \text { for } i=1, \ldots, m \text { implies } x \approx y, \\
& d_{i}(x, x) \approx 0 \quad \text { for } i=1, \ldots, m, \\
& q(x, y, 0,0, \ldots, 0) \approx 0, \\
& q\left(x, y, y, d_{1}(x, y), \ldots, d_{m}(x, y)\right) \approx x
\end{aligned}
$$

hold in $\mathscr{V}$.

(4) The mapping from $\operatorname{Con}(\mathbf{A}) \longrightarrow \mathrm{I}(\mathbf{A})$ defined by $\theta \longmapsto 0 / \theta$ is a lattice isomorphism.

THEOREM 2.7. For a variety $\mathscr{V}$ the following are equivalent.

(1) $\mathscr{V}$ is clot determined.

(2) For all $\mathbf{A} \in \mathscr{V}$ and $S \in \mathrm{Sc}(\mathbf{A})$, we have $S=(0 / S)^{*}$.

(3) $\mathscr{V}$ is ideal determined and has permutable congruences.

(4) $\mathscr{V}$ is 0-regular and has permutable congruences.

(5) For every $\mathbf{A} \in \mathscr{V}, \mathbf{A}$ is 0 -regular and $\operatorname{Con}(\mathbf{A})=\operatorname{Sc}(\mathbf{A})$.

(6) There exist a natural number $m$, binary terms $d_{1}(x, y), \ldots, d_{m}(x, y)$ and an $(m+2)$-term $q$ such that

$$
\begin{aligned}
& d_{i}(x, x) \approx 0 \text { for } i=1, \ldots, m, \\
& q(x, y, 0, \ldots, 0) \approx x, \\
& q\left(x, y, d_{1}(x, y), \ldots, d_{m}(x, y)\right) \approx y
\end{aligned}
$$

hold in $\mathscr{V}$.

(7) The mapping from $\mathrm{Sc}(\mathbf{A}) \longrightarrow \mathrm{Cl}(\mathbf{A})$ defined by $S \longmapsto 0 / S$ is a lattice isomorphism. 
Proof. First we show that (1), (2) and (7) are equivalent. Assume (1); then the mapping $S \longmapsto 0 / S$ is one-to-one and it is also onto by Proposition 2.2. Moreover it is order preserving and its inverse $X \longmapsto X^{*}$ is also order preserving. These are well-known to be sufficient conditions for a mapping between lattices to be an isomorphism. Hence (1) implies (7). It is straightforward to check that (7) implies (2) and (2) implies (1), and hence they are equivalent. From the fact that in a congruence permutable variety any semicongruence is a congruence [12], it follows that (3) implies (1) and the equivalence of (3), (4) and (5) as well.

Let us then show that (1) implies (6). Consider once again $\mathbf{F}_{\mathscr{V}}(x, y)$, and let $M=\{d(x, y): d(x, x)=0\}$. For $d \in M$, let $S_{d}$ be the semicongruence generated by the single pair $\langle 0, d(x, y)\rangle$ and let $S_{M}=\bigvee_{d \in M} S_{d}$. Let $f$ be the endomorphism of $\mathbf{F}_{\mathscr{V}}(x, y)$ defined by $f(x)=f(y)=x, f(0)=0$. Let $S_{f}$ be the semicongruence generated by $\{\langle 0, u\rangle: f(u)=0\}$, i.e. $S_{f}=$ $\left(f^{-1}(0)\right)^{*}$.

Claim. $S_{f}$ is the semicongruence $R$ generated by $\langle x, y\rangle$.

It is easily seen that $f^{-1}(0)$ is a clot and hence $0 / S_{f}=f^{-1}(0)$ by Proposition 2.2. Let $\langle 0, h\rangle \in R$; then for a suitable term $t$ and for $\mathbf{u} \in \mathbf{F}_{\mathscr{V}}(x, y)$ we have $0=t(\mathbf{u}, x, \ldots, x)$ and $h=t(\mathbf{u}, y, \ldots, y)$. Then

$$
\begin{aligned}
f(h) & =t(\overrightarrow{f(u)}, f(y), \ldots \varphi(y)) \\
& =t(\overrightarrow{f(u)}, x, \ldots, x) \\
& =f(t(\mathbf{u}, x, \ldots, x))=f(0)=0,
\end{aligned}
$$

that is, $h \in f^{-1}(0)$. Conversely if $f(u)=0$, say $u=u(x, y)$, then $0=$ $f(u)=f(d(x, y))=d(x, x)$ and hence $\langle 0, u\rangle=\langle d(x, x), d(x, y)\rangle=$ $d(\langle x, x\rangle,\langle x, y\rangle) \in R$. Hence the claim is proved.

With an argument close to the one above we can show that $S_{M}=S_{f}$ and hence $S_{M}$ is a compact in $\operatorname{Sc}(\mathbf{A})$. Then there are $d_{1}, \ldots, d_{m} \in M$ with

$$
S_{M}=S_{d_{1}} \vee \cdots \vee S_{d_{M}}=\left\langle\left\{\left\langle 0, d_{i}(x, y)\right\rangle: i=1, \ldots, m\right\}\right\rangle \text { Sc. }
$$

Now, since $\langle x, y\rangle \in\left\langle\left\{\left\langle 0, d_{i}(x, y)\right\rangle: i=1, \ldots, m\right\}\right\rangle$ Sc , the usual Mal'cev argument yields the existence of a term $q$ satisfying the identities in (6).

Assume now (6). If we define

$$
t(x, y, z)=q\left(x, z, d_{1}(y, z), \ldots, d_{m}(y, z)\right)
$$

it is clear that $t(x, y, z)$ satisfies the Mal'cev identities for permutability. Moreover if $d_{i}(x, y)=0$ for all $i \leq m$, then $x=y$, and hence $\mathscr{V}$ is 0 -regular [4]. So (6) implies (4) and the proof is finished. 
Finally let us quote two examples showing that the classes of varieties defined by Theorems $2.4,2.6$ and 2.7 are distinct. The variety of implication algebras is a variety with one binary operation satisfying

$$
(x y) x \approx x, \quad(x y) y=(y x) x, \quad x(y z) \approx y(x z) .
$$

It has been observed in [5] that this variety, with regard to the equationally definable constant $x x$, is ideal determined but it is not congruence permutable (see also [8]). Hence it satisfies the equivalent conditions of Theorem 2.6 but not of Theorem 2.7.

For the other counterexample, let $\mathbf{A}=\langle\{0,1,2,3,4,5\}, s, 0\rangle$, where $s$ is binary and defined as

$$
s(x, y)= \begin{cases}x & \text { if } y=0 \\ 0 & \text { otherwise. }\end{cases}
$$

It is clear that $\mathbf{A}$ generates a 0 -permutable variety and that every partition of $A$ to which $\{0\}$ belongs is in fact a congruence. If the variety generated by $\mathbf{A}$ were ideal determined, then it would be also congruence modular [5], but this is not the case since $\operatorname{Con}(\mathbf{A})$ is not modular, as the reader can easily check. Hence it satisfies the equivalent conditions of Theorem 2.4 but not of Theorem 2.6.

\section{References}

[1] P. Agliano, 'The prime spectrum of a universal algebra', Rivista di Mat. Pura ed Appl., 4 (1989), 83-91.

[2] P. Agliano and A. Ursini, 'Cosets in universal algebra', J. of Algebra, 107 (1987), 376384.

[3] P. Agliano and A. Ursini, 'On some ideal basis theorems', Rapporto Matematico n.182, Universitá di Siena (1988).

[4] K. Fichtner, 'Eine bemerkung über mannigfaltigkeiten universeller algebren mit idealen', Monatsch. d. Deutsch. Akad. d. Wissen (Berlin) 12 (1970), 21-45.

[5] H.P. Gumm and A. Ursini, 'Ideals in Universal Algebra', Algebra Universalis 19 (1984), 45-54.

[6] A.I. Mal'cev, 'On the general theory of algebraic systems', Mat. Sborsnik 77 (1954), 3-20.

[7] R. McKenzie, G. McNulty and W. Taylor, Algebras, lattices, varietie, Volume I, Wadsworth and Brooks Cole, Monterey, California, 1987.

[8] A. Mitschke, 'Implication algebras are 3-permutable and 3-distributive', Algebra Universalis 1 (1971), 182-186.

[9] A. Ursini, 'Sulle varietá di algebre con una buona teoria degli ideali', Boll. U.M.I. 6 (1972), 90-95.

[10] A. Ursini, 'Osservazioni sulla varietá BIT', Boll, U.M.I. 8 (1973), 205-211. 
[11] A. Ursini, 'Ideals and their Calculus I', Rapporto Matematico n.41, Universitá di Siena, (1981).

[12] H. Werner, 'A Mal'cev condition for admissible relations', Algebra Universalis 3 (1973), 263.

Dipartimento di Matematica

Universitá di Siena

Via del Capitano

53100 Siena, Italy 